\title{
ENDOMORPHISM ALGEBRAS OF 2-TERM SILTING COMPLEXES
}

\author{
ASLAK BAKKE BUAN AND YU ZHOU
}

\begin{abstract}
Aвstract. We study possible values of the global dimension of endomorphism algebras of 2-term silting complexes. We show that for any algebra $A$ whose global dimension gl.dim $A \leq 2$ and any 2-term silting complex $\mathbf{P}$ in the bounded derived category $D^{b}(A)$ of $A$, the global dimension of $\operatorname{End}_{D^{b}(A)}(\mathbf{P})$ is at most 7. We also show that for each $n>2$, there is an algebra $A$ with $\operatorname{gl} \cdot \operatorname{dim} A=n$ such that $D^{b}(A)$ admits a 2-term silting complex $\mathbf{P}$ with gl.dim $\operatorname{End}_{D^{b}(A)}(\mathbf{P})$ infinite.
\end{abstract}

\section{INTRODUCTION}

Let $A$ be a finite dimensional algebra over a field $k$. Let $T$ be a (classical) tilting module in the category $\bmod A$ of finite dimensional right $A$-modules; that is the projective dimension $\operatorname{pd} T$ is at most 1, we have $\operatorname{Ext}_{A}^{1}(T, T)=0$ and there is an exact sequence $0 \rightarrow A \rightarrow T_{1} \rightarrow T_{2} \rightarrow 0$ with $T_{1}, T_{2}$ in add $T$, the additive closure of $T$. Let $B=\operatorname{End}_{A}(T)$. Then, it is a well-known fact (see for example [8, III, Section 3.4] for a more general statement) that $\operatorname{gl} \operatorname{dim} B \leq \operatorname{gl} . \operatorname{dim} A+1$, where gl.dim $A$ denotes the global dimension of $A$.

In this paper we investigate to which extent this generalizes to the following setting. We now consider a 2-term silting complex $\mathbf{P}$ in the bounded homotopy category of finitely generated projective $A$-modules, $K^{b}(\operatorname{proj} A)$. This is just a map between projective $A$-modules, considered as a complex, with the property that $\operatorname{Hom}_{K^{b}(\operatorname{proj} A)}(\mathbf{P}, \mathbf{P}[1])=0$ where [1] denotes the shift functor, and such that $\mathbf{P}$ generates $K^{b}(\operatorname{proj} A)$ as a triangulated category. Note that $K^{b}(\operatorname{proj} A)$ can be considered to be a full triangulated subcategory of the derived category $D^{b}(A)$.

The concept of silting complexes originated from [11], and has more recently been studied by many authors, often motivated by combinatorial aspects related to mutations, as in [2]. Moreover, the case of 2-term silting is of particular interest, see e.g. [1], [4] and [12].

In the setting of 2-term silting, we have the following theorem:

Theorem 0.1. Let $B=\operatorname{End}_{D^{b}(A)}(\mathbf{P})$, for a 2-term silting complex $\mathbf{P}$ in $K^{b}(\operatorname{proj} A)$. Then the following hold.

(a) If $\operatorname{gl} \cdot \operatorname{dim} A=1$, then $\operatorname{gl} \cdot \operatorname{dim} B \leq 3$.

(b) If gl.dim $A=2$, then gl.dim $B \leq 7$.

Moreover, for each $n>2$, there is an algebra $A$, with $\operatorname{gl} \cdot \operatorname{dim} A=n$, such that $K^{b}(\operatorname{proj} A)$ admits $a$ 2-term silting complex $\mathbf{P}$ with gl.dim $\operatorname{End}_{D^{b}(A)}(\mathbf{P})=\infty$.

Note that the projective presentation of a tilting $A$-module $T$ as defined above, gives rise to a 2-term silting complex $\mathbf{P}_{T}$ in $K^{b}(\operatorname{proj} A)$, and that we have an isomorphism of algebras $\operatorname{End}_{A}(T) \cong$ $\operatorname{End}_{D^{b}(A)}\left(\mathbf{P}_{T}\right)$.

The situation in part (a) was studied in [6]. In this case $B$ is a called a silted algebra, and it was proved that silted algebras are so-called shod algebras [7], in particular this implies that gl.dim $B \leq 3$, by [9].

This work was supported by FRINAT grant number 231000, from the Norwegian Research Council. Support by the Institut Mittag-Leffler (Djursholm, Sweden) is gratefully acknowledged. 
The main body of this paper is a proof of (b), an example that the global dimension of $B$ actually can be 7 in this case, and a class of examples that justifies the last statement of Theorem 0.1

We also prove that with a stronger assumption on $\mathbf{P}$, we actually get that $\operatorname{gl} . \operatorname{dim} B$ is bounded by $\operatorname{gl} . \operatorname{dim} A$. More precisely, we show the following.

Theorem 0.2. With the above notation, and assuming in addition that $\mathrm{pd} H^{0}(\mathbf{P}) \leq 1$, we have gl.dim $B \leq 2(\operatorname{gl} \cdot \operatorname{dim} A)+2$.

In the first section, we recall some notation and background concerning 2-term silting complexes and their endomorphism algebras. In the second section, we prove some preliminary general results. Then, in Section 3 and 4, we prove respectively Theorem 0.2 and Theorem 0.1 , while in the last section, we give some examples.

\section{BACKGROUND AND NOTATION}

Let $A$ be a finite dimensional algebra with gl.dim $A=d$. Then $K^{b}(\operatorname{proj} A)=D^{b}(A):=\mathcal{D}$. Let $\mathbf{P}$ be a 2-term silting complex in $\mathcal{D}$ and let $B=\operatorname{End}_{\mathcal{D}}(\mathbf{P})$. We recall some classical notation (see e.g. [3]) and some results from [5], which will be used freely in the remaining of the paper.

Recall that a pair of subcategories $(\mathcal{X}, \mathcal{Y})$ of $\bmod A$, is called a torsion pair, if the following hold:

- $\operatorname{Hom}_{A}(X, Y)=0$ if and only if $Y$ is in $\mathcal{Y}$, and

- $\operatorname{Hom}_{A}(X, \mathcal{Y})=0$ if and only if $X$ is in $X$.

For a given torsion pair $(X, Y)$ and an object $M$ in $\bmod A$, there is a (unique) exact sequence

$$
0 \rightarrow t M \rightarrow M \rightarrow M / t M \rightarrow 0
$$

with $t M$ in $X$ and $M / t M$ in $\mathcal{Y}$. This is called the canonical sequence of $M$. Furthermore, for an $A$-module $X$ we let add $X$ denote the additive closure of $X$ in $\bmod A$, and we let Fac $X$ denote the full subcategory of all quotients of modules in add $X$. The first notion is also used for a complex $X$ in $\mathcal{D}$.

For a 2-term silting complex $\mathbf{P}$, consider the full subcategories of $\bmod A$ given by

$-\mathcal{T}(\mathbf{P})=\left\{X \in \bmod A \mid \operatorname{Hom}_{\mathcal{D}}(\mathbf{P}, X[1])=0\right.$, and

- $\mathcal{F}(\mathbf{P})=\left\{Y \in \bmod A \mid \operatorname{Hom}_{\mathcal{D}}(\mathbf{P}, Y)=0\right.$.

Furthermore, let $B=\operatorname{End}_{\mathcal{D}}(\mathbf{P})$. The following summarizes results from [5] which will be essential later in this paper.

Proposition 1.1. Let $\mathbf{P}$ be a 2-term silting complex in $K^{b}(\operatorname{proj} A)$. Then the following hold.

(a) The pair $(\mathcal{T}(\mathbf{P}), \mathcal{F}(\mathbf{P}))$ is a torsion pair in $\bmod A$.

(b) $\mathcal{T}(\mathbf{P})=\operatorname{Fac} H^{0}(\mathbf{P})$.

(c) The category $C(\mathbf{P})=\{\mathbf{X} \in \mathcal{D} \mid \operatorname{Hom}(\mathbf{P}, \mathbf{X}[i])=0$ for $i \neq 0\}$ is an abelian category with short exact sequences coinciding with the triangles in $\mathcal{D}$ whose vertices are in $C(\mathbf{P})$.

(d) Let $\mathbf{X}$ be in $\mathcal{D}$. Then we have that $\mathbf{X}$ is in $C(\mathbf{P})$ if and only if $H^{0}(\mathbf{X})$ is in $\mathcal{T}(\mathbf{P}), H^{-1}(\mathbf{X})$ is in $\mathcal{F}(\mathbf{P})$ and $H^{i}(\mathbf{X})=0$ for $i \neq-1,0$.

(e) $\operatorname{Hom}_{\mathcal{D}}(\mathbf{P},-): C(\mathbf{P}) \rightarrow \bmod B$ is an equivalence of (abelian) categories.

For full subcategories $\mathcal{X}$ and $\mathcal{Y}$ of $\mathcal{D}$, we let $\mathcal{X} * \mathcal{Y}$ denote the full subcategory of $\mathcal{D}$ with objects $Z$ appearing in a triangle

$$
X \rightarrow Z \rightarrow Y \rightarrow X[1]
$$

with $X$ in $X$ and $Y$ in $\mathcal{Y}$. It follows from the octahedral axiom that we have $(X * Y) * \mathcal{Z}=$ $\mathcal{X} *(\mathcal{Y} * \mathcal{Z})$, for three full subcategories $\mathcal{X}, \mathcal{Y}$ and $\mathcal{Z}$. The subcategory $\mathcal{X}$ is called extension 
closed if $\mathcal{X} * \mathcal{X}=\mathcal{X}$. We will need the following fact, which follows from [10, Propositions 2.1 and 2.4].

Lemma 1.2. Let $\mathcal{X}_{i}$ be subcategories of $\mathcal{D}$, with $\operatorname{Hom}_{\mathcal{D}}\left(\mathcal{X}_{i}, \mathcal{X}_{j}\right)=0=\operatorname{Hom}_{\mathcal{D}}\left(\mathcal{X}_{i}, \mathcal{X}_{j}[1]\right)$ for $i<j$. Then $\mathcal{X}_{1} * \mathcal{X}_{2} * \cdots * \mathcal{X}_{n}$ is closed under extensions and direct summands.

\section{Preliminaries}

Now, fix a 2-term silting complex $\mathbf{P}$ in $K^{b}(\operatorname{proj} A)$, and let $\mathcal{P}=\operatorname{add} \mathbf{P}$. In this section we include some general observations on projective objects and projective dimensions in $C(\mathbf{P})$.

For each $\mathbf{P}_{0}$ in $\mathcal{P}$, given by $P_{0}^{-1} \stackrel{p_{0}}{\rightarrow} P_{0}^{0}$, consider the canonical exact sequence of $H^{-1}\left(\mathbf{P}_{0}\right)$ relative to the torsion pair $(\mathcal{T}(\mathbf{P}), \mathcal{F}(\mathbf{P}))$ :

$$
0 \rightarrow t H^{-1}\left(\mathbf{P}_{0}\right) \rightarrow H^{-1}\left(\mathbf{P}_{0}\right) \rightarrow H^{-1}\left(\mathbf{P}_{0}\right) / t H^{-1}\left(\mathbf{P}_{0}\right) \rightarrow 0 .
$$

So $t H^{-1}\left(\mathbf{P}_{0}\right)$ is a submodule of $P_{0}^{-1}$ and we denote by $\pi: P_{0}^{-1} \rightarrow P_{0}^{-1} / t H^{-1}\left(\mathbf{P}_{0}\right)$ the canonical epimorphism. Let $\widetilde{\mathbf{P}}_{0}$ be the complex $P_{0}^{-1} / t H^{-1}\left(\mathbf{P}_{0}\right) \stackrel{\widetilde{p}_{0}}{\rightarrow} P_{0}^{0}$, where $\widetilde{p}_{0}$ is the unique homomorphism such that the diagram

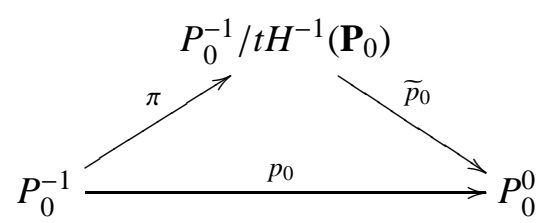

commutes.

Let $\mathcal{P}_{C}=\mathcal{P} \cap C(\mathbf{P})$.

Lemma 2.1. Let $\mathbf{P}_{0}$ be in $\mathcal{P}$. Then $\mathbf{P}_{0}$ is in $\mathcal{P}_{C}$ if and only if $\mathbf{P}_{0} \cong \widetilde{\mathbf{P}}_{0}$.

Proof. We have by definition that $\mathbf{P}_{0} \cong \widetilde{\mathbf{P}}_{0}$ if and only if $t H^{-1}\left(\mathbf{P}_{0}\right)=0$ if and only if $H^{-1}\left(\mathbf{P}_{0}\right)$ is in $\mathcal{F}(\mathbf{P})$ if and only if $\operatorname{Hom}\left(\mathbf{P}, H^{-1}\left(\mathbf{P}_{0}\right)\right)=0$. It is straightforward to check that $\operatorname{Hom}\left(\mathbf{P}, H^{-1}\left(\mathbf{P}_{0}\right)\right)=$ 0 if and only if $\operatorname{Hom}\left(\mathbf{P}, \mathbf{P}_{0}[-1]\right)=0$. Moreover, we have that $\operatorname{Hom}\left(\mathbf{P}, \mathbf{P}_{0}[-1]\right)=0$ if and only if $\mathbf{P}_{0}$ is in $C(\mathbf{P})$, and the statement follows from this.

Lemma 2.2. With notation as above, the following hold.

(a) There is a triangle in $\mathcal{D}$ :

$$
t H^{-1}(\mathbf{P})[1] \rightarrow \mathbf{P} \rightarrow \widetilde{\mathbf{P}} \rightarrow t H^{-1}(\mathbf{P})[2] .
$$

(b) The object $\widetilde{\mathbf{P}}$ is a projective generator for $C(\mathbf{P})$.

Proof. The triangle in (a) exists by the construction of $\widetilde{\mathbf{P}}$.

Note that $H^{0}(\widetilde{\mathbf{P}})=H^{0}(\mathbf{P})$ is in $\mathcal{T}(\mathbf{P})$ and $H^{-1}(\widetilde{\mathbf{P}})=H^{-1}(\mathbf{P}) / t H^{-1}(\mathbf{P})$ is in $\mathcal{F}(\mathbf{P})$. Then by Proposition 1.1(d), we have $\widetilde{\mathbf{P}} \in \mathcal{C}(\mathbf{P})$. Applying the functor $\operatorname{Hom}_{\mathcal{D}}(\mathbf{P},-)$ to this triangle yields an isomorphism

$$
\operatorname{Hom}_{\mathcal{D}}(\mathbf{P}, \mathbf{P}) \cong \operatorname{Hom}_{\mathcal{D}}(\mathbf{P}, \widetilde{\mathbf{P}})
$$

as $B$-modules. Now (b) follows from Proposition $1.1(\mathrm{e})$.

For any integer $i$, we let $\mathcal{D}^{\leq i}(\mathbf{P})=\left\{\mathbf{X} \in \mathcal{D} \mid \operatorname{Hom}_{\mathcal{D}}(\mathbf{P}, \mathbf{X}[j])=0\right.$ for $\left.j>i\right\}$, and we let $\mathcal{D}^{\geq i}(\mathbf{P})=\left\{\mathbf{X} \in \mathcal{D} \mid \operatorname{Hom}_{\mathcal{D}}(\mathbf{P}, \mathbf{X}[j])=0\right.$ for $\left.j<i\right\}$.

Lemma 2.3. With notation as above, we have: $\mathcal{C}(\mathbf{P}) \subset \mathcal{P} * \mathcal{P}[1] * \cdots * \mathcal{P}[d+1]$. 
Proof. By [2, Proposition 2.23], we have

$$
\mathcal{C}(\mathbf{P}) \subset \mathcal{D}^{\leq 0}(\mathbf{P}) \subset \mathcal{P} * \mathcal{P}[1] * \cdots * \mathcal{P}[l-1] * \mathcal{P}[l]
$$

for some $l>0$. For any $\mathbf{M}$ in $C(\mathbf{P})$, by Proposition $1.1(\mathrm{~d})$, we have $H^{i}(\mathbf{M})=0$ for $i \neq-1,0$. So there is a complex $\mathbf{X}$ of projective $A$-modules, which is equivalent to $\mathbf{M}$, and such that $H^{i}(\mathbf{X})=0$ for $i>0$ or $i<-d-1$. So

$$
\operatorname{Hom}_{\mathcal{D}}(\mathbf{M}, \mathbf{P}[i]) \cong \operatorname{Hom}_{\mathcal{D}}(\mathbf{X}, \mathbf{P}[i])=0, i \geq d+2,
$$

which implies that $\mathbf{M}$ is in $\mathcal{P} * \mathcal{P}[1] * \cdots * \mathcal{P}[d+1]$.

Lemma 2.4. For a complex $\mathbf{X}$ in $C(\mathbf{P}) \cap\left(\mathcal{P}_{C} * \mathcal{P}_{C}[1] * \cdots \mathcal{P}_{C}[m]\right)$ for some $m \geq 0$, we have $\operatorname{pd} \operatorname{Hom}_{\mathcal{D}}(\mathbf{P}, \mathbf{X})_{B} \leq m$.

Proof. Let $\mathbf{X}_{0}=\mathbf{X}$. There are triangles

$$
\mathbf{X}_{i+1} \rightarrow \mathbf{O}_{i} \stackrel{g_{i}}{\rightarrow} \mathbf{X}_{i} \rightarrow \mathbf{X}_{i+1}[1], \quad 0 \leq i \leq m-1
$$

where $\mathbf{O}_{i}$ is in $\mathcal{P}_{C}$ and $\mathbf{X}_{i}$ is in $\mathcal{P}_{C} * \mathcal{P}_{C}[1] * \cdots * \mathcal{P}_{C}[m-i]$. Since $\operatorname{Hom}_{\mathcal{D}}(\mathbf{P}, \mathbf{P}[i])=0$ for all

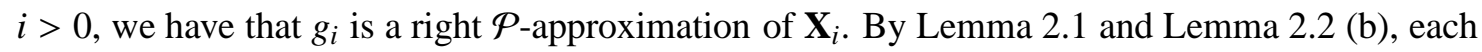
$\mathbf{O}_{i}$ is projective in $C(\mathbf{P})$. Assume that $\mathbf{X}_{i}$ is in $C(\mathbf{P})$ for some $0 \leq i \leq m-1$. Then, since $g_{i}$ is a right $\mathcal{P}$-approximation and $\mathbf{O}_{i}$ is projective in $C(\mathbf{P})$, we have that $g_{i}$ is an epimorphism in $C(\mathbf{P})$. So $\mathbf{X}_{i+1}$ is the kernel of $g_{i}$, by Proposition 1.1 (c). Note that $\mathbf{X}_{0} \in C(\mathbf{P})$. Then by induction on $i$, we have that $\mathbf{X}_{i} \in C(\mathbf{P})$ for all $0 \leq i \leq m$ and

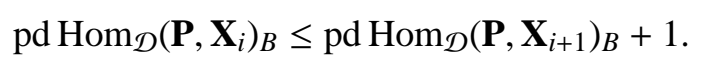

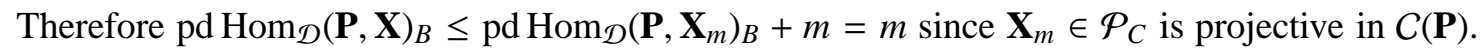

We end this section by considering the following special case. Recall from [13], that a 2-term silting complex $\mathbf{P}$ in $K^{b}(\operatorname{proj} A)$ is a tilting complex if $\operatorname{Hom}_{\mathcal{D}}(\mathbf{P}, \mathbf{P}[-1])=0$.

Proposition 2.5. If the 2-term silting complex $\mathbf{P}$ is a tilting complex, then $\operatorname{gl} \cdot \operatorname{dim} \operatorname{End}_{\mathcal{D}}(\mathbf{P}) \leq$ gl.dim $A+1$.

Proof. If $\mathbf{P}$ is tilting, then $\mathbf{P}$ is in $C(\mathbf{P})$. So we infer that $\mathcal{P}=\mathcal{P}_{C}$. It follows from Lemma2.3 and Lemma 2.4 that gl.dim $\operatorname{End}_{\mathcal{D}}(\mathbf{P}) \leq \operatorname{gl} . \operatorname{dim} A+1$.

Note that the classical situation (as in [8, III, section 3.4]) where $\mathbf{P}$ is the projective resolution of a classical tilting module, is covered by this result.

\section{The partial tilting CASE}

Throughout this section, we assume that $\operatorname{pd}^{0}(\mathbf{P})_{A} \leq 1$, that is: $H^{0}(\mathbf{P})$ is a partial tilting $A$ module. Then we have that $Q=H^{-1}(\mathbf{P})$ is projective as an $A$-module, and $\mathbf{P} \cong H^{0}(\mathbf{P}) \oplus Q[1]$. Consider the canonical exact sequence of $Q$ relative to the torsion pair $(\mathcal{T}(\mathbf{P}), \mathcal{F}(\mathbf{P}))$ :

$$
0 \rightarrow t Q \rightarrow Q \rightarrow Q / t Q \rightarrow 0 \text {. }
$$

As before, we let $d=\operatorname{gl}$.dim $A$. We first prove two technical lemmas.

Lemma 3.1. With the above notation, we have

$$
t Q \in \operatorname{add} H^{0}(\mathbf{P}) * \operatorname{add} H^{0}(\mathbf{P})[1] * \cdots * \operatorname{add} H^{0}(\mathbf{P})[d-1] .
$$


Proof. We first note that $t Q \in \mathcal{T}(\mathbf{P})$, so by definition $\operatorname{Hom}_{D^{b}(A)}(\mathbf{P}, t Q[i])=0$ for $i \neq 0$. In particular, we have $\operatorname{Hom}_{D^{b}(A)}(Q[1], t Q[i])=0$ for $i \neq 0$. For $i=0$, since both $Q$ and $t Q$ are $A$-modules, we also have that $\operatorname{Hom}_{D^{b}(A)}(Q[1], t Q)=0$. It follows from $T(\mathbf{P}) \in C(\mathbf{P})$ that by Proposition 1.1, we have $t Q \in \mathcal{P} * \mathcal{P}[1] * \cdots * \mathcal{P}[d+1]$. Therefore, using $\mathbf{P} \cong H^{0}(\mathbf{P}) \oplus Q[1]$, we get that $t Q$ is in add $H^{0}(\mathbf{P}) *$ add $H^{0}(\mathbf{P})[1] * \cdots * \operatorname{add} H^{0}(\mathbf{P})[d+1]$. By the canonical sequence of $Q$, we have $\operatorname{pd}(t Q)_{A} \leq \operatorname{pd}(Q / t Q)_{A}-1 \leq d-1$. Hence, it follows that $\operatorname{Hom}(t Q, \mathbf{P}[d])=0=$ $\operatorname{Hom}(t Q, \mathbf{P}[d+1])$. The claim of the lemma follows.

Lemma 3.2. With the above notation, we have $C(\mathbf{P}) \subset \mathcal{P} * \mathcal{P}[1] * \cdots * \mathcal{P}[d] * \operatorname{add} H^{0}(\mathbf{P})[d+1]$.

Proof. Using that $\mathbf{P} \cong H^{0}(\mathbf{P}) \oplus Q[1]$ in combination with Lemma 2.3, we only need to prove that $\operatorname{Hom}_{\mathcal{D}}(\mathbf{X}, Q[d+2])=0$ for $\mathbf{X} \in C(\mathbf{P})$. This follows from $\operatorname{pd} H^{i}(\mathbf{X})_{A} \leq d$ for $i=-1,0$ and $H^{i}(\mathbf{X})=0$ for $i \neq-1,0$.

We can now prove the main result of this section.

Theorem 3.3. If $\operatorname{pd}\left(H^{0}(\mathbf{P})\right)_{A} \leq 1$, then $\operatorname{gl} \operatorname{dim} B \leq 2 \mathrm{gl} \cdot \operatorname{dim} A+2$.

Proof. Let $\mathbf{X}$ be an object in $C(\mathbf{P})$ with

$$
\mathbf{X} \in \mathcal{P} * \cdots * \mathcal{P}[i] * \text { add } H^{0}(\mathbf{P})[i+1] * \cdots * \operatorname{add} H^{0}(\mathbf{P})[d+1]
$$

for some $0 \leq i \leq d$. Then there is a triangle

$$
\mathbf{X}_{1} \rightarrow \mathbf{E} \stackrel{g_{\mathbf{X}}}{\rightarrow} \mathbf{X} \rightarrow \mathbf{X}_{1}[1]
$$

where $g_{\mathbf{X}}$ is a right $\mathcal{P}$-approximation of $\mathbf{X}$ and $\mathbf{X}_{1}$ is in

$$
\mathcal{P} * \cdots * \mathcal{P}[i-1] * \operatorname{add} H^{0}(\mathbf{P})[i] * \cdots * \operatorname{add} H^{0}(\mathbf{P})[d] .
$$

Then $\operatorname{Hom}_{\mathcal{D}}\left(\mathbf{P}, g_{\mathbf{X}}\right)$ is an epimorphism and $\operatorname{Hom}_{\mathcal{D}}(\mathbf{P}, \mathbf{E})$ is projective in $\bmod B$.

Recall that $Q=H^{-1}(\mathbf{P})$. Then, by Lemma2.2 there is a triangle

$$
F[1] \rightarrow \mathbf{E} \rightarrow \widetilde{\mathbf{E}} \rightarrow F[2]
$$

where $F$ is in add $t Q \subset \mathcal{T}(\mathbf{P}) \subset C(\mathbf{P})$ and $\widetilde{\mathbf{E}}$ is projective in $C(\mathbf{P})$. So $\operatorname{Hom}_{\mathcal{D}}(F[1], \mathbf{X})=0$ since $\mathbf{X} \in C(\mathbf{P})$. It follows that the map $g_{\mathbf{X}}$ factors through the map $\mathbf{E} \rightarrow \widetilde{\mathbf{E}}$. Then, by the octahedral axiom, we have the following commutative diagram of triangles:

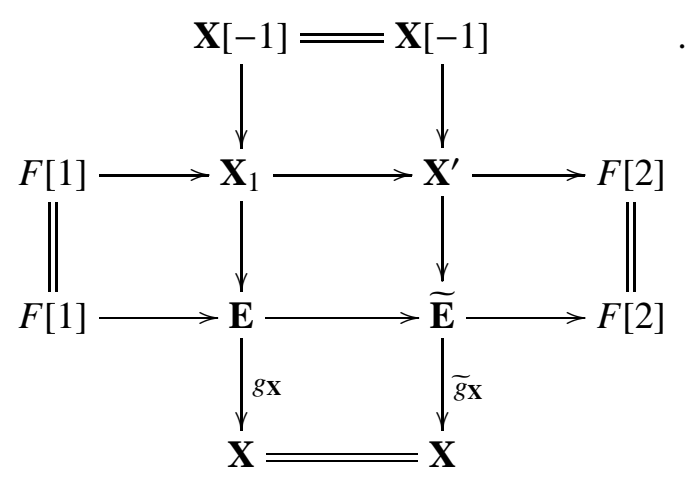

Then we have that

$$
\begin{aligned}
\mathbf{X}^{\prime} \in & \operatorname{add} \mathbf{X}_{1} * \operatorname{add} F[2] \\
\subset & \left(\mathcal{P} * \cdots * \mathcal{P}[i-1] * \operatorname{add} H^{0}(\mathbf{P})[i] * \cdots * \operatorname{add} H^{0}(\mathbf{P})[d]\right) \\
& *\left(\operatorname{add} H^{0}(\mathbf{P}) * \cdots * \operatorname{add} H^{0}(\mathbf{P})[d-1]\right)[2] \\
= & \left(\mathcal{P} * \cdots * \mathcal{P}[i-1] * \operatorname{add} H^{0}(\mathbf{P})[i] * \cdots * \operatorname{add} H^{0}(\mathbf{P})[d]\right) * \operatorname{add} H^{0}(\mathbf{P})[d+1]
\end{aligned}
$$


where the inclusion is due to Lemma 3.1, and the equality follows from

$$
\mathcal{P} * \cdots * \mathcal{P}[i-1] * \text { add } H^{0}(\mathbf{P})[i] * \cdots * \operatorname{add} H^{0}(\mathbf{P})[d]
$$

being closed under extensions by Lemma 1.2 Applying $\operatorname{Hom}_{\mathcal{D}}(\mathbf{P},-)$ to the above diagram, we obtain a commutative diagram

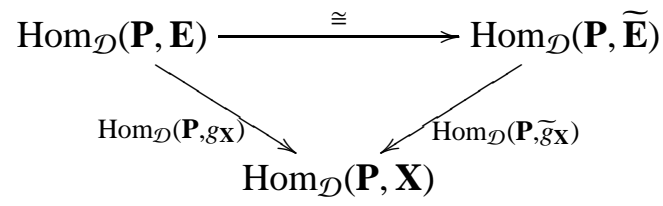

Using that the map $\operatorname{Hom}_{\mathcal{D}}\left(\mathbf{P}, g_{\mathbf{X}}\right)$ is an epimorphism in $\bmod B$, it follows that the map $\widetilde{g}_{\mathbf{X}}$ is an epimorphism in $C(\mathbf{P})$. Then $\mathbf{X}^{\prime}$ is the kernel of $\widetilde{g}_{\mathbf{X}}$ in $C(\mathbf{P})$. Hence

$$
\operatorname{pd~} \operatorname{Hom}_{\mathcal{D}}(\mathbf{P}, \mathbf{X})_{B} \leq \operatorname{pd} \operatorname{Hom}_{\mathcal{D}}\left(\mathbf{P}, \mathbf{X}^{\prime}\right)_{B}+1 .
$$

Using induction on $i$ and Lemma 3.2, we have that for $\mathbf{X} \in C(\mathbf{P})$, there is $\mathbf{X}^{\prime}$ such that

$$
\mathbf{X}^{\prime} \in C(\mathbf{P}) \cap\left(\operatorname{add} H^{0}(\mathbf{P}) * \operatorname{add} H^{0}(\mathbf{P})[1] * \cdots * \operatorname{add} H^{0}(\mathbf{P})[d+1]\right)
$$

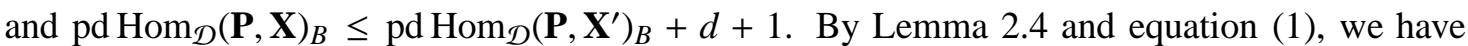
$\operatorname{pd} \operatorname{Hom}_{\mathcal{D}}\left(\mathbf{P}, \mathbf{X}^{\prime}\right)_{B} \leq d+1$. It then follows that $\operatorname{pd} \operatorname{Hom}_{\mathcal{D}}(\mathbf{P}, \mathbf{X})_{B} \leq 2 d+2$, and hence gl.dim $B \leq$ $2 d+2$.

\section{The CASE OF GLOBAL Dimension 2.}

In this section, we consider the case when $\operatorname{gl} \operatorname{dim} A \leq 2$. Our aim is to prove part (b) of Theorem 0.1 stating that in this case we have that the global dimension is at most 7 for the endomorphism algebra of any 2-term silting complex.

We prepare by showing four technical lemmas. Let $\mathcal{P}_{C}^{[0,1]}=(\mathcal{P} * \mathcal{P}[1]) \cap C(\mathbf{P})$.

Lemma 4.1. If $\mathbf{X}$ is in $\mathcal{P}_{C}^{[0,1]}$, then $\operatorname{pd}^{H_{0}}{ }_{\mathcal{D}}(\mathbf{P}, \mathbf{X})_{B} \leq 1$.

Proof. Since $\mathbf{X}$ is in $\mathcal{P} * \mathcal{P}$ [1], there is a triangle $\mathbf{O}_{1} \rightarrow \mathbf{O}_{0} \rightarrow \mathbf{X} \rightarrow \mathbf{O}_{1}[1]$ with $\mathbf{O}_{0}, \mathbf{O}_{1} \in \mathcal{P}$. Applying the functor $\operatorname{Hom}_{\mathcal{D}}(\mathbf{P},-)$ to this triangle, we get a long exact sequence

$$
\operatorname{Hom}_{\mathcal{D}}(\mathbf{P}, \mathbf{X}[-1]) \rightarrow \operatorname{Hom}_{\mathcal{D}}\left(\mathbf{P}, \mathbf{O}_{1}\right) \rightarrow \operatorname{Hom}_{\mathcal{D}}\left(\mathbf{P}, \mathbf{O}_{0}\right) \rightarrow \operatorname{Hom}_{\mathcal{D}}(\mathbf{P}, \mathbf{X}) \rightarrow \operatorname{Hom}_{\mathcal{D}}\left(\mathbf{P}, \mathbf{O}_{1}[1]\right)
$$

where the first term is zero since $\mathbf{X}$ is in $C(\mathbf{P})$, and the last term is zero since $\operatorname{Hom}_{\mathcal{D}}(\mathbf{P}, \mathbf{P}[1])=0$.

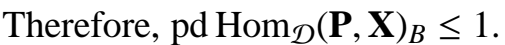

Lemma 4.2. If $\mathbf{X}$ is in $\mathcal{C}(\mathbf{P}) \cap\left(\mathcal{P}_{C}^{[0,1]} * \mathcal{P}_{C}^{[0,1]}[1] * \mathcal{P}_{C}^{[0,1]}[2]\right)$, then $\operatorname{pd} \operatorname{Hom}_{\mathcal{D}}(\mathbf{P}, \mathbf{X})_{B} \leq 3$.

Proof. By $\mathbf{X} \in \mathcal{P}_{C}^{[0,1]} * \mathcal{P}_{C}^{[0,1]}[1] * \mathcal{P}_{C}^{[0,1]}[2]$, there are triangles

$$
\mathbf{L} \rightarrow \mathbf{D}_{1} \rightarrow \mathbf{X} \rightarrow \mathbf{L}[1]
$$

and

$$
\mathbf{D}_{3} \rightarrow \mathbf{D}_{2} \rightarrow \mathbf{L} \rightarrow \mathbf{D}_{3}[1]
$$

with $\mathbf{D}_{1}, \mathbf{D}_{2}, \mathbf{D}_{3} \in \mathcal{P}_{C}^{[0,1]}$ and $\mathbf{L} \in \mathcal{P}_{C}^{[0,1]} * \mathcal{P}_{C}^{[0,1]}[1] \subset \mathcal{P} * \mathcal{P}[1] * \mathcal{P}[2]$. Applying $\operatorname{Hom}_{\mathcal{D}}(\mathbf{P},-)$ to triangle (2), we obtain a long exact sequence

$$
\begin{aligned}
\operatorname{Hom}_{\mathcal{D}}(\mathbf{P}, \mathbf{X}[-2]) \rightarrow \operatorname{Hom}_{\mathcal{D}}(\mathbf{P}, \mathbf{L}[-1]) \rightarrow \operatorname{Hom}_{\mathcal{D}}\left(\mathbf{P}, \mathbf{D}_{1}[-1]\right) \rightarrow \operatorname{Hom}_{\mathcal{D}}(\mathbf{P}, \mathbf{X}[-1]) \\
\rightarrow \operatorname{Hom}_{\mathcal{D}}(\mathbf{P}, \mathbf{L}) \rightarrow \operatorname{Hom}_{\mathcal{D}}\left(\mathbf{P}, \mathbf{D}_{1}\right) \rightarrow \operatorname{Hom}_{\mathcal{D}}(\mathbf{P}, \mathbf{X}) \rightarrow \operatorname{Hom}_{\mathcal{D}}(\mathbf{P}, \mathbf{L}[1]) .
\end{aligned}
$$


We have $\operatorname{Hom}_{\mathcal{D}}(\mathbf{P}, \mathbf{X}[i])=0$ for $i=-1$ or $i=-2$, since $\mathbf{X}$ is in $C(\mathbf{P})$. Furthermore, we have $\operatorname{Hom}_{\mathcal{D}}\left(\mathbf{P}, \mathbf{D}_{1}[-1]\right)=0$, by $\mathbf{D}_{1} \in C(\mathbf{P})$ and $\operatorname{Hom}_{\mathcal{D}}(\mathbf{P}, \mathbf{L}[1])=0$ by $\mathbf{L} \in \mathcal{P} * \mathcal{P}[1] * \mathcal{P}[2]$. From this it follows that we have a short exact sequence

$$
0 \rightarrow \operatorname{Hom}_{\mathcal{D}}(\mathbf{P}, \mathbf{L}) \rightarrow \operatorname{Hom}_{\mathcal{D}}\left(\mathbf{P}, \mathbf{D}_{1}\right) \rightarrow \operatorname{Hom}_{\mathcal{D}}(\mathbf{P}, \mathbf{X}) \rightarrow 0
$$

and that $\operatorname{Hom}_{\mathcal{D}}(\mathbf{P}, \mathbf{L}[-1])=0$. Using this short exact sequence, it follows that

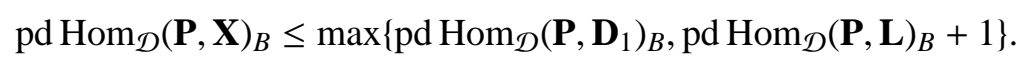

Applying $\operatorname{Hom}_{\mathcal{D}}(\mathbf{P},-)$ to the triangle (3), we obtain an exact sequence

$$
0=\operatorname{Hom}_{\mathcal{D}}(\mathbf{P}, \mathbf{L}[-1]) \rightarrow \operatorname{Hom}_{\mathcal{D}}\left(\mathbf{P}, \mathbf{D}_{3}\right) \rightarrow \operatorname{Hom}_{\mathcal{D}}\left(\mathbf{P}, \mathbf{D}_{2}\right) \rightarrow \operatorname{Hom}_{\mathcal{D}}(\mathbf{P}, \mathbf{L}) \rightarrow \operatorname{Hom}_{\mathcal{D}}\left(\mathbf{P}, \mathbf{D}_{3}[1]\right)
$$

where the last term is zero due to $\mathbf{D}_{3} \in \mathcal{P}_{C}^{[0,1]}$. As above, we obtain that

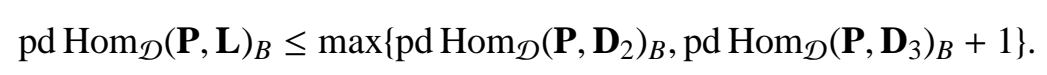

Now, combining the inequalities (4) and (5) with Lemma 4.1, we obtain $\operatorname{pd} \operatorname{Hom}_{\mathcal{D}}(\mathbf{P}, \mathbf{X})_{B} \leq$ 3.

Lemma 4.3. If $\mathbf{N}$ is in $\mathcal{D}^{\geq-1}(\mathbf{P}) \cap(\mathcal{P} * \mathcal{P}[1] * \mathcal{P}[2])$, then there is an object $\widetilde{\mathbf{N}} \in \mathcal{C}(\mathbf{P})$ such that $\operatorname{Hom}_{\mathcal{D}}(\mathbf{P}, \mathbf{N}) \cong \operatorname{Hom}_{\mathcal{D}}(\mathbf{P}, \widetilde{\mathbf{N}})$ as $B$-modules and $\widetilde{\mathbf{N}} \in \operatorname{add} \mathbf{N} * \mathcal{P}_{C}^{[0,1]}[2]$.

Proof. Since $\left(\mathcal{D}^{\leq 0}(\mathbf{P}), \mathcal{D}^{\geq 0}(\mathbf{P})\right)$ is a $t$-structure (see [12, Lemma 5.10]), there is a triangle

$$
\mathbf{M} \rightarrow \mathbf{N} \rightarrow \widetilde{\mathbf{N}} \rightarrow \mathbf{M}[1]
$$

with $\mathbf{M} \in \mathcal{D}^{\leq 0}(\mathbf{P})[1]$ and $\widetilde{\mathbf{N}} \in \mathcal{D}^{\geq 0}(\mathbf{P})$. Then $\mathbf{M} \in \mathcal{P}[1] * \cdots * \mathcal{P}[l]$ for some $l$ by [2, Proposition 2.23]. Applying the functor $\operatorname{Hom}_{\mathcal{D}}(\mathbf{P},-)$ to the triangle [6], we have a long exact sequence

$$
\cdots \rightarrow \operatorname{Hom}_{\mathcal{D}}(\mathbf{P}, \mathbf{M}[i]) \rightarrow \operatorname{Hom}_{\mathcal{D}}(\mathbf{P}, \mathbf{N}[i]) \rightarrow \operatorname{Hom}_{\mathcal{D}}(\mathbf{P}, \widetilde{\mathbf{N}}[i]) \rightarrow \operatorname{Hom}_{\mathcal{D}}(\mathbf{P}, \mathbf{M}[i+1]) \rightarrow \cdots
$$

Since $\operatorname{Hom}_{\mathcal{D}}(\mathbf{P}, \mathbf{M}[i])=0$ for $i \geq 0$ by $\mathbf{M} \in \mathcal{P}[1] * \cdots * \mathcal{P}[l]$ and $\operatorname{Hom}_{\mathcal{D}}(\mathbf{P}, \widetilde{\mathbf{N}}[i])=0$ for $i<0$ by $\widetilde{\mathbf{N}} \in \mathcal{D}^{\geq 0}(\mathbf{P})$, and also $\operatorname{Hom}_{\mathcal{D}}(\mathbf{P}, \mathbf{N}[i])=0$ for $i \neq-1,0$ by the assumption $\mathbf{N} \in$ $\mathcal{D}^{\geq-1}(\mathbf{P}) \cap(\mathcal{P} * \mathcal{P}[1] * \mathcal{P}[2])$, we have that

$$
\operatorname{Hom}_{\mathcal{D}}(\mathbf{P}, \mathbf{N}) \cong \operatorname{Hom}_{\mathcal{D}}(\mathbf{P}, \widetilde{\mathbf{N}})
$$

as $B$-modules,

$$
\operatorname{Hom}_{\mathcal{D}}(\mathbf{P}, \widetilde{\mathbf{N}}[i])=0 \text {, for } i>0,
$$

and

$$
\operatorname{Hom}_{\mathcal{D}}(\mathbf{P}, \mathbf{M}[i])=0 \text {, for } i<-1 \text {. }
$$

Thus, we obtain $\widetilde{\mathbf{N}} \in \mathcal{D}^{\leq 0}(\mathbf{P}) \cap \mathcal{D}^{\geq 0}(\mathbf{P})=C(\mathbf{P})$ and $\mathbf{M} \in \mathcal{D}^{\geq 0}(\mathbf{P})[1] \cap \mathcal{D}^{\leq 0}(\mathbf{P})[1]=C(\mathbf{P})[1]$. Then by Lemma 2.3, we have $\widetilde{\mathbf{N}} \in \mathcal{P} * \mathcal{P}[1] * \mathcal{P}[2] * \mathcal{P}[3]$. Applying the functor $\operatorname{Hom}_{\mathcal{D}}(-, \mathbf{P})$ to the triangle (6), we obtain a long exact sequence

$$
\cdots \rightarrow \operatorname{Hom}_{\mathcal{D}}(\widetilde{\mathbf{N}}, \mathbf{P}[i]) \rightarrow \operatorname{Hom}_{\mathcal{D}}(\mathbf{N}, \mathbf{P}[i]) \rightarrow \operatorname{Hom}_{\mathcal{D}}(\mathbf{M}, \mathbf{P}[i]) \rightarrow \operatorname{Hom}_{\mathcal{D}}(\widetilde{\mathbf{N}}, \mathbf{P}[i+1]) \rightarrow \cdots
$$

We have $\operatorname{Hom}_{\mathcal{D}}(\mathbf{N}, \mathbf{P}[i])=0$ for $i>2$ by $\mathbf{N} \in \mathcal{P} * \mathcal{P}[1] * \mathcal{P}[2]$, and we have $\operatorname{Hom}_{\mathcal{D}}(\widetilde{\mathbf{N}}, \mathbf{P}[i])=0$ for $i>3$ by $\widetilde{\mathbf{N}} \in \mathcal{P} * \mathcal{P}[1] * \mathcal{P}[2] * \mathcal{P}[3]$. From this it follows that $\operatorname{Hom}_{\mathcal{D}}(\mathbf{M}, \mathbf{P}[i])=0$ for $i>2$, and hence $\mathbf{M} \in(\mathscr{P}[1] * \mathcal{P}[2]) \cap \mathcal{C}(\mathbf{P})[1]=\mathcal{P}_{C}^{[0,1]}[1]$. Therefore we have that

$$
\widetilde{\mathbf{N}} \in \operatorname{add} \mathbf{N} * \operatorname{add} \mathbf{M}[1] \subset \text { add } \mathbf{N} * \mathcal{P}_{C}^{[0,1]}[2] .
$$


Lemma 4.4. Let $\mathbf{X} \in C(\mathbf{P}) \cap(\mathcal{P} * \mathcal{P}[1] * \cdots * \mathcal{P}[t] * \mathcal{H}[t+1])$ for some $t$ with $0 \leq t \leq 3$, where $\mathcal{H} \subset \mathcal{P} * \mathcal{P}[1] * \cdots * \mathcal{P}[2-t]$ (and $\mathcal{H}=0$ for $t=3$ ). Then for each $r$ with $0 \leq r \leq \min \{t+1,3\}$, there is an object $\widetilde{\mathbf{X}}_{r} \in C(\mathbf{P})$ such that

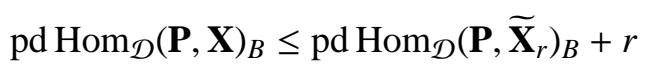

and

$$
\widetilde{\mathbf{X}}_{r} \in \mathcal{P} * \mathcal{P}[1] * \cdots * \mathcal{P}[t-r] * \mathcal{H}[t+1-r] * \mathcal{P}_{C}^{[0,1]}[3-r] * \cdots * \mathcal{P}_{C}^{[0,1]}[2]
$$

where $\mathcal{P} * \mathcal{P}[1] * \cdots * \mathcal{P}[t-r]$ is taken to be 0 when $r=t+1$ and $\mathcal{P}_{C}^{[0,1]}[3-r] * \cdots * \mathcal{P}_{C}^{[0,1]}[2]$ is taken to be 0 when $r=0$.

Proof. Let $\widetilde{\mathbf{X}}_{0}=\mathbf{X}$. Then $\widetilde{\mathbf{X}}_{0}$ satisfies the conditions in the lemma. Assume that $\widetilde{\mathbf{X}}_{r-1}$ satisfying the conditions. By

$$
\widetilde{\mathbf{X}}_{r-1} \in \mathcal{P} * \mathcal{P}[1] * \cdots * \mathcal{P}[t-(r-1)] * \mathcal{H}[t+1-(r-1)] * \mathcal{P}_{C}^{[0,1]}[3-(r-1)] * \cdots * \mathcal{P}_{C}^{[0,1]}[2],
$$

there is a triangle

$$
\mathbf{X}_{r} \rightarrow \mathbf{P}_{0} \rightarrow \widetilde{\mathbf{X}}_{r-1} \rightarrow \mathbf{X}_{r}[1]
$$

with $\mathbf{P}_{0} \in \mathcal{P}, \mathbf{X}_{r} \in \mathcal{P} * \cdots * \mathcal{P}[t-r] * \mathcal{H}[t+1-r] * \mathcal{P}_{C}^{[0,1]}[3-r] * \cdots * \mathcal{P}_{C}^{[0,1]}[1] \subset \mathcal{P} * \mathcal{P}[1] * \mathcal{P}[2]$. The inclusion follows from Lemma 1.2. Applying $\operatorname{Hom}_{\mathcal{D}}(\mathbf{P},-)$ to this triangle, we have a long exact sequence

$$
\cdots \rightarrow \operatorname{Hom}_{\mathcal{D}}\left(\mathbf{P}, \mathbf{X}_{r}[i]\right) \rightarrow \operatorname{Hom}_{\mathcal{D}}\left(\mathbf{P}, \mathbf{P}_{0}[i]\right) \rightarrow \operatorname{Hom}_{\mathcal{D}}\left(\mathbf{P}, \widetilde{\mathbf{X}}_{r-1}[i]\right) \rightarrow \operatorname{Hom}_{\mathcal{D}}\left(\mathbf{P}, \mathbf{X}_{r}[i+1]\right) \rightarrow \cdots
$$

Since $\operatorname{Hom}_{\mathcal{D}}\left(\mathbf{P}, \widetilde{\mathbf{X}}_{r-1}[i]\right)=0$ for $i \neq 0$ by $\widetilde{\mathbf{X}}_{r-1} \in \mathcal{C}(\mathbf{P}), \operatorname{Hom}_{\mathcal{D}}\left(\mathbf{P}, \mathbf{X}_{r}[1]\right)=0$ by $\mathbf{X}_{r} \in \mathcal{P} * \mathcal{P}[1] *$ $\mathcal{P}[2]$, and also $\operatorname{Hom}_{\mathcal{D}}\left(\mathbf{P}, \mathbf{P}_{0}[i]\right)=0$ for $i<-1$ by $\mathbf{P}$ being 2 -term, we have a short exact sequence

$$
0 \rightarrow \operatorname{Hom}_{\mathcal{D}}\left(\mathbf{P}, \mathbf{X}_{r}\right) \rightarrow \operatorname{Hom}_{\mathcal{D}}\left(\mathbf{P}, \mathbf{P}_{0}\right) \rightarrow \operatorname{Hom}_{\mathcal{D}}\left(\mathbf{P}, \widetilde{\mathbf{X}}_{r-1}\right) \rightarrow 0
$$

and

$$
\operatorname{Hom}_{\mathcal{D}}\left(\mathbf{P}, \mathbf{X}_{r}[i]\right)=0 \text { for } i<-1 .
$$

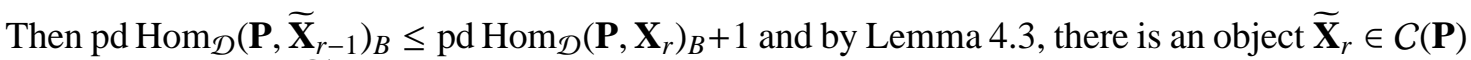
such that $\operatorname{Hom}_{\mathcal{D}}\left(\mathbf{P}, \widetilde{\mathbf{X}}_{r}\right)_{B} \cong \operatorname{Hom}_{\mathcal{D}}\left(\mathbf{P}, \mathbf{X}_{r}\right)_{B}$ and

$\widetilde{\mathbf{X}}_{r} \in \operatorname{add} \mathbf{X}_{r} * \mathcal{P}_{C}^{[0,1]}[2] \subset \mathcal{P} * \mathcal{P}[1] * \cdots * \mathcal{P}[t-r] * \mathcal{H}[t+1-r] * \mathcal{P}_{C}^{[0,1]}[3-r] * \cdots * \mathcal{P}_{C}^{[0,1]}[1] * \mathcal{P}_{C}^{[0,1]}[2]$.

Now we prove the main result in this section.

Theorem 4.5. If $\operatorname{gl} . \operatorname{dim} A \leq 2$, then $\operatorname{gl} . \operatorname{dim} \operatorname{End}_{\mathcal{D}}(\mathbf{P}) \leq 7$ for any 2-term silting complex $\mathbf{P}$ in $K^{b}(\operatorname{proj} A)$

Proof. Let $\mathbf{X} \in \mathcal{C}(\mathbf{P})$. Then by Lemma 2.3, we have that $\mathbf{X} \in \mathcal{P} * \mathcal{P}[1] * \mathcal{P}[2] * \mathcal{P}[3]$. By Lemma 4.4, (taking $t=3, r=2$ and hence $\mathcal{H}=0$ ), there is an $\widetilde{\mathbf{X}} \in \mathcal{C}(\mathbf{P})$ such that $\widetilde{\mathbf{X}} \in$ $\mathcal{P} * \mathcal{P}[1] * \mathcal{P}_{C}^{[0,1]}[1] * \mathcal{P}_{C}^{[0,1]}[2]$, and

$$
\operatorname{pd} \operatorname{Hom}_{\mathcal{D}}(\mathbf{P}, \mathbf{X})_{B} \leq \operatorname{pd} \operatorname{Hom}_{\mathcal{D}}(\mathbf{P}, \widetilde{\mathbf{X}})_{B}+2 .
$$

Then there is a triangle

$$
\mathbf{Z} \rightarrow \mathbf{Y} \rightarrow \widetilde{\mathbf{X}} \rightarrow \mathbf{Z}[1]
$$

with $\mathbf{Y} \in \mathcal{P} * \mathcal{P}[1]$ and $\mathbf{Z} \in \mathcal{P}_{C}^{[0,1]} * \mathcal{P}_{C}^{[0,1]}[1]$. Applying the functor $\operatorname{Hom}_{\mathcal{D}}(\mathbf{P},-)$ to this triangle, we have a long exact sequence

$$
\cdots \rightarrow \operatorname{Hom}_{\mathcal{D}}(\mathbf{P}, \mathbf{Z}[i]) \rightarrow \operatorname{Hom}_{\mathcal{D}}(\mathbf{P}, \mathbf{Y}[i]) \rightarrow \operatorname{Hom}_{\mathcal{D}}(\mathbf{P}, \widetilde{\mathbf{X}}[i]) \rightarrow \operatorname{Hom}_{\mathcal{D}}(\mathbf{P}, \mathbf{Z}[i+1]) \rightarrow \cdots
$$


Since $\operatorname{Hom}_{\mathcal{D}}(\mathbf{P}, \widetilde{\mathbf{X}}[i])=0$ for $i \neq 0$ by $\widetilde{\mathbf{X}} \in \mathcal{C}(\mathbf{P})$, and $\operatorname{Hom}_{\mathcal{D}}(\mathbf{P}, \mathbf{Z}[i])=0$ for $i \neq-1,0$ by $\mathbf{Z} \in \mathcal{C}(\mathbf{P}) * C(\mathbf{P})[1]$, we have a short exact sequence

$$
0 \rightarrow \operatorname{Hom}_{\mathcal{D}}(\mathbf{P}, \mathbf{Z}) \rightarrow \operatorname{Hom}_{\mathcal{D}}(\mathbf{P}, \mathbf{Y}) \rightarrow \operatorname{Hom}_{\mathcal{D}}(\mathbf{P}, \widetilde{\mathbf{X}}) \rightarrow 0
$$

and

$$
\operatorname{Hom}_{\mathcal{D}}(\mathbf{P}, \mathbf{Y}[i])=0 \text { for } i<-1 .
$$

Then we have that

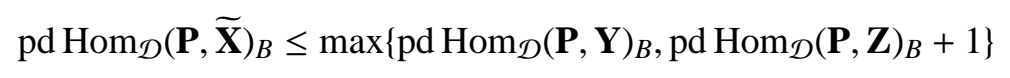

and $\mathbf{Y}, \mathbf{Z} \in \mathcal{D}^{\geq-1}(\mathbf{P})$. By Lemma 4.3, there are objects $\widetilde{\mathbf{Y}}, \widetilde{\mathbf{Z}} \in C(\mathbf{P})$ such that:

$$
\begin{array}{ll}
\widetilde{\mathbf{Y}} \in \mathcal{P} * \mathcal{P}[1] * \mathcal{P}_{C}^{[0,1]}[2] & \widetilde{\mathbf{Z}} \in \mathcal{P}_{C}^{[0,1]} * \mathcal{P}_{C}^{[0,1]}[1] * \mathcal{P}_{C}^{[0,1]}[2] \\
\operatorname{Hom}_{\mathcal{D}}\left(\mathbf{P}, \widetilde{\mathbf{Y}}_{B}\right) \cong \operatorname{Hom}_{\mathcal{D}}(\mathbf{P}, \mathbf{Y})_{B} & \operatorname{Hom}_{\mathcal{D}}(\mathbf{P}, \widetilde{\mathbf{Z}})_{B} \cong \operatorname{Hom}_{\mathcal{D}}(\mathbf{P}, \mathbf{Z})_{B}
\end{array}
$$

By Lemma 4.4, (taking $t=1, r=2$ and $\mathcal{H}=\mathcal{P}_{C}^{[0,1]}[2]$ ), there is an object $\widetilde{\mathbf{Y}^{\prime}} \in \mathcal{C}(\mathbf{P})$ such that $\widetilde{\mathbf{Y}^{\prime}} \in \mathcal{P}_{\mathcal{C}}^{[0,1]} * \mathcal{P}_{\mathcal{C}}^{[0,1]}[1] * \mathcal{P}_{\mathcal{C}}^{[0,1]}[2]$ and

$$
\operatorname{pd} \operatorname{Hom}_{\mathcal{D}}(\mathbf{P}, \widetilde{\mathbf{Y}})_{B} \leq \operatorname{pd} \operatorname{Hom}_{\mathcal{D}}\left(\mathbf{P}, \widetilde{\mathbf{Y}^{\prime}}\right)_{B}+2 .
$$

By Lemma 4.2, we have $\operatorname{pd}^{\operatorname{Hom}_{\mathcal{D}}}(\mathbf{P}, \widetilde{\mathbf{Z}})_{B} \leq 3$ and $\operatorname{pd}_{\operatorname{Hom}}\left(\mathbf{P}, \widetilde{\mathbf{Y}^{\prime}}\right)_{B} \leq 3$. Hence, combining (7), (8) and (9), we obtain

$$
\begin{aligned}
{\operatorname{pd~} \operatorname{Hom}_{\mathcal{D}}(\mathbf{P}, \mathbf{X})_{B}} & \leq \operatorname{pd} \operatorname{Hom}_{\mathcal{D}}(\mathbf{P}, \widetilde{\mathbf{X}})_{B}+2 \\
& \leq \max \left\{\operatorname{pd} \operatorname{Hom}_{\mathcal{D}}(\mathbf{P}, \mathbf{Y})_{B}, \operatorname{pd}_{\operatorname{Hom}_{\mathcal{D}}}(\mathbf{P}, \mathbf{Z})_{B}+1\right\}+2 \\
& =\max \left\{\operatorname{pd} \operatorname{Hom}_{\mathcal{D}}(\mathbf{P}, \widetilde{\mathbf{Y}})_{B}, \operatorname{pd}_{\operatorname{Hom}_{\mathcal{D}}}(\mathbf{P}, \widetilde{\mathbf{Z}})_{B}+1\right\}+2 \\
& \leq \max \left\{\operatorname{pd} \operatorname{Hom}_{\mathcal{D}}\left(\mathbf{P}, \widetilde{\mathbf{Y}^{\prime}}\right)_{B}+2, \operatorname{pdom}_{\mathcal{D}}(\mathbf{P}, \widetilde{\mathbf{Z}})_{B}+1\right\}+2 \\
& \leq 7 .
\end{aligned}
$$

\section{EXAMPLES}

5.1. First example. We first give an example to show that the bound in Theorem 3.3 is possible. Let $n \geq 2$ and $A=\mathbf{k} Q / I$ where $Q$ is the following quiver

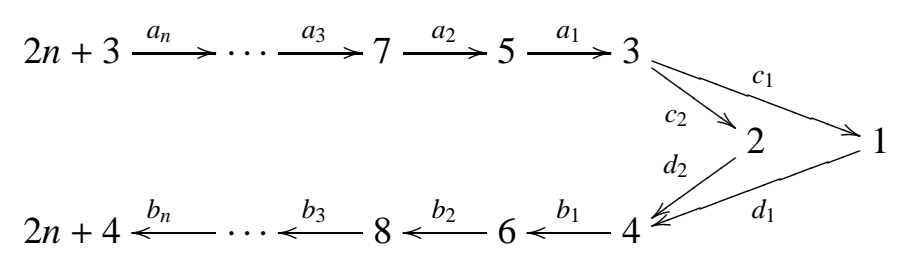

and the ideal $I$ is generated by $c_{1} d_{1}-c_{2} d_{2}, a_{i+1} a_{i}$ and $b_{i} b_{i+1}, 1 \leq i \leq n-1$. Then $\operatorname{gl} \operatorname{dim} A=n$. Let $\mathbf{P}$ be the direct sum of the following complexes in $K^{b}(\operatorname{proj} A)$ :

$$
\begin{array}{ccc}
0 & \longrightarrow & \bigoplus_{1 \leq i \leq n+2, i \neq 2} P_{2 i} \\
P_{4} & \longrightarrow & P_{2} \\
P_{1} & \longrightarrow & P_{3} \\
\bigoplus_{1 \leq i \leq n+2, i \neq 2} P_{2 i-1} & \longrightarrow & 0
\end{array}
$$


It is easily verified that $\mathbf{P}$ is a 2 -term silting complex. The quiver of the endomorphism ring $\operatorname{End}_{\mathcal{D}}(\mathbf{P})$ is the Dynkin quiver of type $A_{2 n+4}$ :

$$
\begin{aligned}
& 2 n+3 \stackrel{a_{n}}{\longrightarrow} \cdots \stackrel{a_{3}}{\longrightarrow} 7 \stackrel{a_{2}}{\longrightarrow} 5 \stackrel{a_{1}}{\longrightarrow} 3 \stackrel{a_{0}}{\longrightarrow} 1 \\
& 2 n+4 \stackrel{b_{n}}{\longleftarrow} \cdots \stackrel{b_{3}}{\longleftarrow} 8 \stackrel{b_{2}}{\longleftarrow} 6 \stackrel{b_{1}}{\longleftarrow} 4 \stackrel{b_{0}}{\longleftarrow} 2^{2}
\end{aligned}
$$

with the relations $a_{i+1} a_{i}=0, b_{i} b_{i+1}=0,1 \leq i \leq n-1$ and $a_{0} c b_{0}=0$. Hence the global dimension of $\operatorname{End}_{\mathcal{D}}(\mathbf{P})$ is $2 n+2$.

5.2. Second example. The next example shows that 7 is a possible value for the global dimension of the endomorphism algebra of a 2-term silting complex over an algebra of global dimension two. Let $A=\mathbf{k} Q / I$ with $Q$ the following quiver

$$
1 \stackrel{a_{1}}{\longrightarrow} 2 \stackrel{a_{2}}{\longrightarrow} 3 \stackrel{a_{3}}{\longrightarrow} 4 \underset{a_{5}}{\stackrel{a_{4}}{\longrightarrow}} 5 \stackrel{a_{6}}{\longrightarrow} 6 \stackrel{a_{7}}{\longrightarrow} 7 \stackrel{a_{8}}{\longrightarrow} 8
$$

and $I$ the ideal generated by $a_{1} a_{2}, a_{3} a_{4} a_{6}$ and $a_{7} a_{8}$. Then $A$ has global dimension two, and the complex $\mathbf{P}$ given by the direct sum of the complexes

$$
\begin{array}{ccc}
0 & \longrightarrow & \bigoplus_{i=5,7,8} P_{i} \\
P_{6} & \longrightarrow & P_{5} \\
P_{4} & \longrightarrow & P_{3} \\
\bigoplus_{i=1,2,4} P_{i} & \longrightarrow & 0
\end{array}
$$

is a 2-term silting complex. It is easily verified, that the quiver of $\operatorname{End}_{\mathcal{D}}(\mathbf{P})$ is a linearly oriented Dynkin quiver of type $A_{8}$ and the ideal of relation equals the square of the Jacobson radical. Hence the global dimension of $\operatorname{End}_{\mathcal{D}}(\mathbf{P})$ is 7 .

5.3. Third example. The last example shows that there is no bound on the global dimension of the endomorphism algebra of a 2-silting object over an algebra with global dimension $d \geq 3$. This example then completes the proof of Theorem 0.1 .

Let first $A=\mathbf{k} Q / I$ where the quiver $Q$ is

$$
3 \underset{b}{\stackrel{a}{\rightleftarrows}} 2 \underset{\left.\right|^{c}}{\stackrel{e}{\rightleftarrows}} 4
$$

and $I=\langle b a, b d, a b c, d e\rangle$. The indecomposable projective $A$-modules are

$$
P_{1}=1, \quad P_{2}=1 \frac{2}{2} 4, \quad P_{3}=\underset{1}{3}, \quad P_{4}=\underset{1}{2} \underset{2}{2} 4 .
$$

The integers here denote the corresponding simples, and the notation indicates the radical filtration. The global dimension of $A$ is 3 . Let $\mathbf{P}$ be the direct sum of

$$
\mathbf{P}_{i}=\cdots \rightarrow 0 \rightarrow P_{i} \rightarrow 0 \rightarrow 0 \rightarrow \cdots, \quad i=1,3,4,
$$

(concentrated in degree -1) and

$$
\mathbf{P}_{2}=\cdots \rightarrow 0 \rightarrow P_{1} \oplus P_{3} \oplus P_{4} \stackrel{p}{\rightarrow} P_{2} \rightarrow 0 \rightarrow \cdots
$$

where $p$ is a projective presentation of $S_{2}$. Then it is easily verified that $\mathbf{P}$ is a 2-term silting complex. 
By Proposition 1.1 (b) we have that $\mathcal{T}(\mathbf{P})=$ Fac $H^{0}(\mathbf{P})$, and hence $\mathcal{T}(\mathbf{P})=$ add $S_{2}$. We will show the projective dimension of $S_{2}$ in $C(\mathbf{P})$ is infinite, by proving that its third syzygy equals $S_{2}$. This implies that a minimal projective resolution of $S_{2}$ is periodic and hence infinite.

Using the notation in Section 2 , we have that $\widetilde{\mathbf{P}}_{1}=\mathbf{P}_{1}$ and $\widetilde{\mathbf{P}}_{3}=\mathbf{P}_{3}$. Moreover $\widetilde{\mathbf{P}}_{4}=\left(P_{4} / S_{2}\right)[1]$, and $\widetilde{\mathbf{P}}_{2}$ is given by the complex

$$
\cdots \rightarrow 0 \rightarrow P_{1} \oplus P_{3} \oplus\left(P_{4} / S_{2}\right) \stackrel{\widetilde{p}}{\rightarrow} P_{2} \rightarrow 0 \rightarrow \cdots
$$

Consider now the triangle

$$
\operatorname{cone}(\pi)[-1] \rightarrow \widetilde{\mathbf{P}}_{2} \stackrel{\pi}{\rightarrow} S_{2} \rightarrow \operatorname{cone}(\pi)
$$

where $\pi$ is

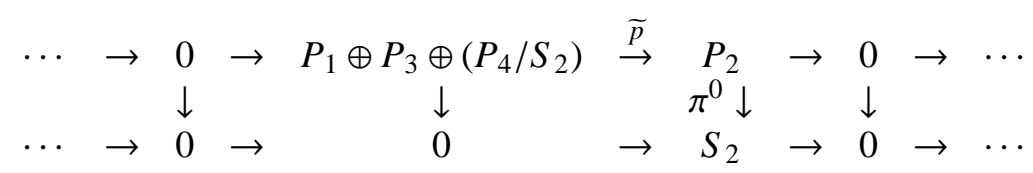

with $\pi^{0}$ being a projective cover of $S_{2}$ in $\bmod A$.

Then $H^{0}(\operatorname{cone}(\pi)[-1])=0, H^{-1}(\operatorname{cone}(\pi)[-1]) \cong H^{-1}\left(\widetilde{\mathbf{P}}_{2}\right) \in \mathcal{F}(\mathbf{P})$ and $H^{i}(\operatorname{cone}(\pi)[-1])=0$ for $i \neq-1,0$. So cone $(\pi)[-1]$ is in $C(\mathbf{P})$, using Proposition 1.1 (d). Hence $\pi$ is a projective cover of $S_{2}$ in $C(\mathbf{P})$ and cone $(\pi)[-1]$ is its kernel in $C(\mathbf{P})$.

Note that $\operatorname{cone}(\pi)[-1] \cong H^{-1}(\operatorname{cone}(\pi)[-1]) \cong H^{-1}\left(\widetilde{\mathbf{P}}_{2}\right) \cong P_{1} \oplus M$, where $M={ }_{13}^{2}{ }_{4} \in \mathcal{F}(\mathbf{P})$. Consider the triangle

$$
\operatorname{cone}\left(\pi_{1}\right)[-1] \rightarrow \widetilde{\mathbf{P}}_{1} \oplus \widetilde{\mathbf{P}}_{3} \oplus \widetilde{\mathbf{P}}_{4} \stackrel{\pi_{1}}{\rightarrow} M[1] \rightarrow \operatorname{cone}\left(\pi_{1}\right)
$$

where $\pi_{1}$ is

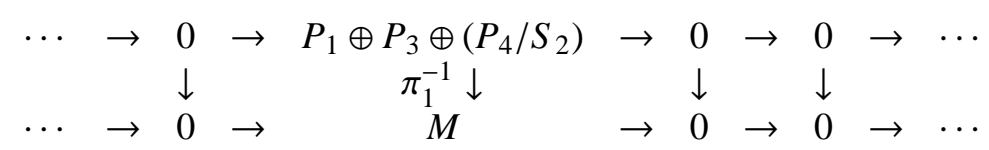

with $\pi_{1}^{-1}$ being the unique (up to a scalar) right minimal homomorphism from $P_{1} \oplus P_{3} \oplus\left(P_{4} / S_{2}\right)$ to $M$. Then $H^{0}\left(\operatorname{cone}\left(\pi_{1}\right)[-1]\right) \cong S_{2} \in \mathcal{T}(\mathbf{P}), H^{-1}\left(\operatorname{cone}\left(\pi_{1}\right)[-1]\right) \cong{ }_{1}^{2} \oplus M \in \mathcal{F}(\mathbf{P})$ (since $\left.\operatorname{Hom}_{A}\left(S_{2}, \frac{2}{1}\right)=0\right)$ and $H^{i}\left(\operatorname{cone}\left(\pi_{1}\right)[-1]\right)=0$ for $i \neq-1,0$. So cone $\left(\pi_{1}\right)[-1] \in C(\mathbf{P})$, hence $\pi_{1}$ is a projective cover of $M[1]$ in $C(\mathbf{P})$ and cone $\left(\pi_{1}\right)[-1]$ is its kernel.

Now consider the triangle

$$
\operatorname{cone}\left(\pi_{2}\right)[-1] \rightarrow \widetilde{\mathbf{P}}_{2} \stackrel{\pi_{2}}{\rightarrow} \operatorname{cone}\left(\pi_{1}\right)[-1] \rightarrow \operatorname{cone}\left(\pi_{2}\right)
$$

where $\pi_{2}$ is

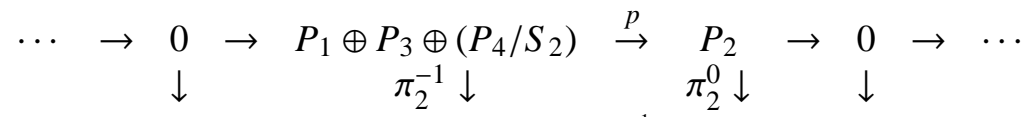

$$
\begin{aligned}
& \cdots \rightarrow 0 \rightarrow P_{1} \oplus P_{3} \oplus\left(P_{4} / S_{2}\right) \stackrel{\pi_{1}^{-1}}{\rightarrow} \quad M \quad \rightarrow \quad 0 \rightarrow \ldots
\end{aligned}
$$

with $\pi_{2}^{-1}$ being the identity map and $\pi_{2}^{0}$ being a projective cover of $M$ in $\bmod A$. Then we have $H^{0}\left(\operatorname{cone}\left(\pi_{2}\right)[-1]\right) \cong S_{2} \in \mathcal{T}(\mathbf{P})$ and $H^{i}\left(\operatorname{cone}\left(\pi_{1}\right)[-1]\right)=0$ for $i \neq 0$. Hence cone $\left(\pi_{2}\right)[-1] \cong S_{2} \in$ $C(\mathbf{P})$ and we have a short exact sequence in $C(\mathbf{P})$ :

$$
0 \rightarrow S_{2} \rightarrow \widetilde{\mathbf{P}}_{2} \stackrel{\pi_{2}}{\rightarrow}\left(\operatorname{cone}\left(\pi_{1}\right)[-1] \rightarrow 0 .\right.
$$

Thus, the projective resolution of $S_{2}$ in $C(\mathbf{P})$ is periodic and hence the projective dimension is infinite. Therefore, also the global dimension of $B$ is infinite, by Proposition 1.1(e). 
Now, for any $n$ consider the quiver $Q_{n}$ given by

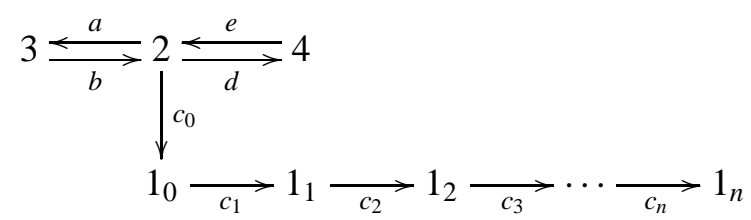

with relations $I_{n}=\left\langle b a, b d, a b c_{0}, d e, c_{0} c_{1}, c_{1} c_{2}, \ldots, c_{n-1} c_{n}\right\rangle$. Consider the algebra $A(n)=k Q_{n} / I_{n}$. We leave it as an exercise to check that $A(n)$ has global dimension $n+3$, and to find a 2-term silting complex $\mathbf{P}^{\prime}$, such that $\operatorname{End}_{D^{b}(A(n))}\left(\mathbf{P}^{\prime}\right)$ has infinite global dimension.

\section{REFERENCES}

[1] T. Adachi, O. Iyama and I. Reiten, $\tau$-tilting theory, Compos. Math. 150 (2015), no. 3, 415-452.

[2] T. Aihara and O. Iyama, Silting mutation in triangulated categories, J. Lond. Math. Soc. (2) 85 (2012), no. 3, 633-668.

[3] I. Assem, D. Simson and A. Skowronski, Elements of the representation theory of associative algebras. Vol. 1. Techniques of Representation Theory, London Mathematical Society Student Texts, 65. Cambridge University Press, Cambridge, 2006.

[4] T. Brüstle and D. Yang, Ordered Exchange Graphs, Advances in representation theory of algebras, 135-193, EMS Ser. Congr. Rep., Eur. Math. Soc., Zürich, 2013.

[5] A. B. Buan and Y. Zhou, A silting theorem, J. Pure Appl. Algebra 220 (2016), no. 7, 2748-2770.

[6] A. B. Buan and Y. Zhou, Silted algebras, preprint arXiv:1506.03649.

[7] F. U. Coelho and M. A. Lanzilotta, Algebras with small homological dimensions, Manuscripta Mathematica 100 (1999) 1-11.

[8] D. Happel, Triangulated categories in the representation theory of finite dimensional algebras, London Mathematical Society Lecture Note Series, 119, Cambridge University Press, Cambridge, 1988.

[9] D. Happel, I. Reiten and S. O. Smalø, Tilting in abelian categories and quasitilted algebra, Mem. Amer. Math. Soc. 120 (1996), no. 575.

[10] O. Iyama and Y. Yoshino, Mutation in triangulated categories and rigid Cohen-Macaulay modules, Invent. Math. 172 (2008), no. 1, 117-168.

[11] B. Keller and D. Vossieck, Aisles in derived categories, Bull. Soc. Math. Belg. Sr. A 40 (1988), no. 2, $239-253$.

[12] S. Koenig and D. Yang, Silting objects, simple-minded collections, $t$-structures and co-t-structures for finitedimensional algebras, Doc. Math. 19 (2014), 403-438.

[13] J. Rickard, Morita theory for derived categories, J. London Math. Soc. (2) 39 (1989), no. 3, 436-456.

Department of Mathematical Sciences Norwegian University of Science and Technology 7491 Trondheim NORWAY

E-mail address: aslakb@math.ntnu.no

Department of Mathematical Sciences Norwegian University of Science and Technology 7491 Trondheim NORWAY

E-mail address: yu.zhou@math.ntnu.no 